Running Head: PARENTAL AUTISTIC TRAITS AND PARENTING DIFFICULTIES

Relationship between Parental Autistic Traits and Parenting Difficulties in a Japanese

\title{
Community Sample
}

Aya Saito ${ }^{1,2,3}$, Satoko Matsumoto ${ }^{2}$, Minori Sato ${ }^{4}$, Yukina Sakata ${ }^{5}$, Hideyuki Haraguchi ${ }^{3}$

${ }^{1}$ Human Science Division, Faculty of Core Research, Ochanomizu University, 2-1-1 Otsuka, Bunkyo-ku, Tokyo, 112-8610, Japan

${ }^{2}$ Institute for Education and Human Development, Ochanomizu University, 2-1-1 Otsuka, Bunkyo-ku, Tokyo, 112-8610, Japan

${ }^{3}$ Department of Preventive Intervention for Psychiatric Disorders, National Institute of Mental Health, National Center of Neurology and Psychiatry (NCNP), 4-1-1 Ogawahigashi, Kodaira, Tokyo 187-8553, Japan

${ }^{4}$ Faculty of Human Sciences and Cultural Studies, Yamanashi Eiwa College, 888, Yokone-machi, Kofu, Yamanashi, 400-8555, Japan

${ }^{5}$ Department of Human and Social Sciences, Faculty of Letters and Education, Ochanomizu University, 2-1-1 Otsuka, Bunkyo-ku, Tokyo, 112-8610, Japan

\begin{abstract}
Author Notes
Correspondence concerning this article should be addressed to Aya Saito; Human Science Division, Faculty of Core Research, Ochanomizu University; 2-1-1 Otsuka, Bunkyo-ku, Tokyo, 112-8610, Japan; Tel: 0081-3-5978-5198; E-mail: saito.aya@ocha.ac.jp
\end{abstract}

Declarations of Interest: The authors declare that they have no known competing financial interests or personal relationships that could have appeared to influence the work reported in this paper. 


\begin{abstract}
Background: Previous studies have suggested an association between higher levels of parental autistic traits and negative aspects of parenting; however, the domain of autistic traits specifically associated with parenting difficulties has not been investigated yet. Moreover, it remains to be determined whether this association exists even after controlling for children's characteristics. Aims: This study examined the relationship between each domain of parental autistic traits and parenting difficulties after controlling for children's characteristics in a Japanese community sample.
\end{abstract}

Methods and Procedures: Surveys were administered to 1,373 Japanese adults who were parents to children in kindergartens, nursery schools, and elementary schools.

Outcomes and Results: The results showed that parents with higher levels of autistic traits had more parenting difficulties. Even after controlling for children's sex, age, and emotional/behavioral problems, paternal impaired communication and maternal poor attention switching, impaired communication, and lack of imagination related to higher parenting difficulties, while a higher maternal level of attention to detail was associated with lower difficulties in parenting.

Conclusions and Implications: The findings of this study highlight the importance of focusing on parental autistic traits and providing appropriate parenting support considering these traits. Keywords: autism spectrum disorder, autistic trait, parenting difficulties, community sample, Autism Quotient 
What this Paper Adds: The results of this study showed a positive association between parental autistic traits and parenting difficulties, consistent with previous research (e.g., Dissanayake et al., 2020), but this study revealed new findings in two aspects mainly. First, even after controlling for the children's characteristics (sex, age, and emotional/behavioral problems), higher levels of parental autistic traits were associated with higher levels of parenting difficulties. The domains of autistic traits associated with parenting difficulties differed between fathers and mothers.

Specifically, the domain of impaired communication was related to parenting difficulties in fathers, whereas the domains of poor attention switching, impaired communication, and lack of imagination were related to parenting difficulties in mothers. These findings suggest the need to focus on parents with higher levels of autistic traits, including those below the diagnostic subthreshold, to prevent or detect early parenting difficulties. In addition, the findings highlight the importance of providing appropriate support for parents with higher autistic traits, to promote social adaptation and the mental health of families, considering the differences between fathers and mothers. 


\section{The Relationship between Parental Autistic Traits and Parenting Difficulties in a Japanese Community Sample}

Autism spectrum disorders (ASDs) are neurodevelopmental disorders characterized by impairment of social communication and interaction across multiple contexts and the existence of restricted and repetitive patterns of behavior, activities, or interests (American Psychiatric Association, 2013). Diagnosis of ASD is not limited to children, and its symptoms often persist into adulthood, with only a few having good outcomes across aspects such as independent living, communication, and learning (Howlin et al., 2004). The combined prevalence of autism in adults of all ages in England was 11/1000 (95\% CI [3/1000, 19/1000; Brugha et al., 2016). With the adoption of the new diagnostic category name, autism spectrum disorder, and the emphasis on the continuity of autistic traits in the Diagnostic and Statistical Manual of Mental Disorders (DSM-5; American Psychiatric Association, 2013), it is now widely recognized that autistic traits exist as a continuum within the general population (Constantino \& Charman, 2016). In Japanese community samples, autistic traits of not only children (Kamio et al., 2013) but also adults have been reported to be continuously distributed (Takei et al., 2014). The prevalence is even higher when adults with higher subthreshold autistic traits are also included for analysis. Furthermore, many adults with higher levels of autistic traits have a family and parent children. Higher levels of autistic traits such as being rigid or poor in social skills and communication can lead to various difficulties in parenting. However, there is still a lack of empirical studies examining the relationship between parental autistic traits and parenting factors. According to van Steijn, Oerlemans, van Aken, et al. (2013), the influence of parental ASD on parenting is largely unknown, most likely because of the sensitive nature of the topic ever since Bettelheim (1967) put forward the idea that autism was caused by a lack of maternal warmth toward children, although this theory is no longer acceptable. 
There are few previous studies on the association between parental autistic traits and parenting. For instance, van Steijn, Oerlemans, de Ruiter, et al. (2013) examined the impact of parental developmental disorder traits, including autistic traits and attention-deficit hyperactivity disorder (ADHD) traits, on parenting style. The parents who participated in the study had two children, one with ASD (with/without ADHD) and a typically developing sibling. The results showed that mothers with higher scores for autistic traits, assessed by the Autism Spectrum Quotient (AQ; Baron-Cohen et al., 2001), as well as fathers with higher ADHD traits, were prone to a more permissive parenting style, which is high in responsiveness but low in demandingness (Baumrind, 1973), only toward their unaffected child. Lau et al. (2016) examined the relationship between autistic traits in parents of children with ASD and parenting efficacy. The results showed that fathers with ASD had the lowest parenting efficacy, while mothers with ASD had comparable levels of parenting efficacy to mothers without ASD diagnosis.

More recently, Dissanayake et al. (2020) conducted a study on the association between parental autistic traits and parenting. They developed the Parenting Needs Questionnaire (PNQ), which measured the level of parenting difficulties, to recognize the needs of parents with higher levels of autistic traits when parenting typically developing children. The results showed that parental autistic traits, measured by AQ (Baron-Cohen et al., 2001), were associated with parenting difficulties, while parenting self-esteem was not uniquely predicted by parental autistic traits, and parents with higher levels of autistic traits tended to have difficulties in various aspects of parenting.

There has been little focus on the association between parental autistic traits and parenting in Japan, apart from two studies that investigated the relationship between maternal autistic traits and child mistreatment. Fujiwara et al. (2014) reported that mothers with higher scores for autistic 
traits, assessed by the self-administered short version of the Pervasive Developmental Disorders Autism Society Japan Rating Scale (PARS; Adachi et al., 2008), were likely to have higher levels of child mistreatment, even after controlling for characteristics at baseline and ADHD traits of mothers. Tachibana et al. (2017) showed higher maternal autistic traits, assessed by the short version of PARS (Adachi et al., 2008), and higher maternal ADHD traits and excessive inhibition of behavior and affect, were risk factors for child maltreatment. Interest in adult ASD has increased significantly in recent years in Japan, and further research on the relationship between autistic traits of parents and parenting behavior is needed.

As mentioned above, some previous studies have suggested an association between parental autistic traits and negative aspects of parenting; however, the domain of autistic traits specifically associated with parenting difficulties has not yet been investigated. There are several dimensions to autistic traits. For instance, the diagnostic criteria for ASD in the DSM-5 (American Psychiatric Association, 2013) include both social communication interaction factors and restricted and repetitive behavior factors. AQ (Baron-Cohen et al., 2001), which is one of the most well-known international rating scales for adult autistic traits, includes five domains: social skills, attention switching, attention to detail, communication, and imagination; however, it is not clear how each of these domains is associated with parenting difficulties.

This study investigated the association between each domain of paternal and maternal autistic traits and parenting difficulties in a Japanese community sample. Since children's emotional/behavioral problems are a factor influencing parenting stress (e.g., McSherry et al., 2019), it was necessary to control these problems in children to more accurately examine the association between parental autistic traits and parenting difficulties. In this study, children's emotional/behavioral problems, assessed using the Strengths and Difficulties Questionnaire 
(SDQ; Goodman, 1997), were controlled to disentangle the effects of parental autistic traits on children's characteristics. By investigating the independent effects of parental autistic traits on parenting difficulties, we could suggest the clinical significance of evaluating parental autistic traits for identifying parents at greater risk of facing parenting difficulties and need appropriate parenting support regardless of their child's characteristics.

\section{Methods}

\subsection{Participants and Procedure}

Data were obtained from a community sample in Japan comprising 428 fathers and 945 mothers. From October 2019 to September 2020, we approached 10 kindergartens and nursery schools, and eight elementary schools in Japan, and all 18 institutions agreed to participate in the survey. Questionnaires (father and mother versions) and explanation sheets were distributed to a total of 4,956 families whose children studied in these 18 institutions via their teachers. In kindergartens and nursery schools, classes for children over two years of age were included in this survey. In elementary schools, all classes from Grades 1 to 6 were included. All children were in regular classes and not in classes for children with disabilities. Each parent completed and voluntarily returned the anonymous questionnaire, which implied their informed consent. Of the 1534 valid responses (528 fathers; 1016 mothers), 161 were excluded for missing one or more items. The final sample comprised 1373 parents (428 fathers; 945 mothers). The average age of the children whose parents provided the data used in this study was 85.12 months ( $S D=34.16$ months), and 699 of the 1373 children were boys.

\subsection{Instruments}

2.2.1. Parental autistic traits. Parental autistic traits were measured using AQ (Baron-Cohen et al., 2001). The AQ is a 50-item quantitative measure of autistic traits. There are 
four choices for the responses of each item: definitely disagree, slightly disagree, slightly agree, and definitely agree. Responses of definitely agree or slightly agree scored 1 point on forward-scored items, while responses of definitely disagree or slightly disagree scored 1 point on reverse-scored items according to the scoring method of the original paper (Baron-Cohen et al., 2001). All 50 items are divided into five subscales (Social Skills, Attention Switching, Attention to Detail, Communication, and Imagination). Each subscale score ranges from 0 to 10 . Higher scores indicate higher levels of autistic traits, that is, higher scores on each subscale indicate lower Social Skills, poor Attention Switching, higher Attention to Detail, impaired Communication, and lack of Imagination, respectively. Cronbach's $\alpha$ in this study was .75 for Social Skills, .57 for Attention Switching, .60 for Attention to Detail, .65 for Communication, and .53 for Imagination. Although the internal consistency is somewhat low, close values have been obtained in previous studies in the non-clinical group (Hurst et al., 2007; Pisula et al., 2013; Ruta et al., 2012). In the present study, we used the Japanese version of the AQ, which each parent rated. Similar to the original version (Baron-Cohen et al., 2001), the Japanese version of the AQ has also been subsequently validated (Wakabayashi et al., 2004). In addition to the total score, the developers of the Japanese version of AQ have stated that each of the five subscale scores can be used to identify which domains are problematic for an individual.

2.2.2. Parenting difficulties. Parenting difficulties scores were measured using PNQ (Dissanayake et al., 2020). The PNQ measures parenting difficulties to reveal the needs of parents with higher levels of autistic traits. It contains 44 parenting behavior items classified into nine subscales (Modeling/Teaching Behaviors, Understanding Needs, Affection, Emotion Control, Attention/Connection, Spontaneity, Communication, Danger Awareness, and Sensory Issues). The sensory issues subscale contains four items, and all other subscales contain five items. Each item is 
scored on a 4-point scale $(0=$ very easy, $1=$ somewhat easy, $2=$ somewhat difficult, $3=$ very difficult). Sensory issues subscale scores range from 0 to 16 , and scores of all other subscales range from 0 to 20. After our research team translated the original version of the PNQ into Japanese with permission from the original author, a specialized translation company performed back translation and asked the original author to confirm the result. In this study, each parent rated their parenting behavior. A higher subscale score means that parenting is more difficult in that domain.

Cronbach's $\alpha$ in this study was .83 for Modeling/Teaching Behaviors (e.g., modeling positive behavior for your child, such as using manners), .77 for Understanding Needs (e.g., understanding the physical needs of your child, such as knowing when s/he is hungry or sleepy), .79 for Affection (e.g., speaking affectionately to your child to express how you feel, such as initiating physical affection with your child), .86 for Emotion Control (e.g., being patient when your child is upset/ crying, for example not getting frustrated if s/he is unsettled), .73 for Attention/Connection (e.g., remaining engaged in social interactions with your child and not becoming distracted), .77 for Spontaneity (e.g., adapting to changes in your child's routine), .73 for Communication (e.g., understanding your child's attempts to verbally communicate something to you), .84 for Danger Awareness (e.g., enforcing rules to keep your child safe), and .83 for Sensory Issues (e.g., overall, coping with how loud your child is).

2.2.3. Emotional/behavioral problems of children. Children's emotional and behavioral problems were measured using the total difficulties score (TDS) of parent-reported SDQ (Goodman, 1997). The SDQ is one of the most widely used scales for measuring emotional/behavioral problems in children. The validity and reliability of the SDQ have been confirmed in a previous study (Goodman, 2001). All 25 items are classified into five subscales: four difficulties subscales (emotional symptoms, conduct problems, hyperactivity/inattention, and 
peer problems) and one prosocial behavior subscale. Each item is scored as 0 (not true), 1 (somewhat true), or 2 (certainly true); thus, each subscale score ranges from 0 to 10. TDS was calculated by adding the scores of the four difficulties subscales, with a range of 0 to 40 . In this study, the TDS reported by mothers was used to measure the total difficulties of children. A higher score indicated more difficulties in emotional/behavioral problems. Cronbach's $\alpha$ for TDS in this study was .78. The validity and reliability of the SDQ in Japanese have also been confirmed in previous research (Matsuishi et al., 2008; Moriwaki \& Kamio, 2014).

\subsection{Ethical Considerations}

This study was approved by the Ethics Committee of [BLINDED FOR REVIEW].

\subsection{Data Analyses}

We calculated correlation coefficients between total and each subscale score for both the AQ and PNQ to investigate the association between parental autistic traits and parenting difficulties. Furthermore, $t$-tests were performed to examine differences in parenting difficulties between parents with high autistic traits and not high autistic traits. Finally, hierarchical multiple regression analyses were performed using the PNQ Total Score as the dependent variable.

Children's sex, age, and TDS were entered as control variables in Step 1. AQ subscale scores of parents were entered in Step 2. Statistical significance was set at $p<.05$. All statistical analyses were conducted using IBM SPSS version 24 (Armonk, NY: IBM Corp.).

\section{Results}

\subsection{Correlational Analyses}

Prior to the correlation analysis, descriptive statistics were calculated to understand the distribution of each variable used in this study (Table 1). Pearson's correlation coefficients were computed to investigate the correlations between the total and subscale scores for parental autistic 
traits and the total and each subscale scores on the PNQ. The total scores of both paternal and maternal autistic traits correlated with the total score of parenting difficulties. Considering each domain of parental autistic traits, both fathers' and mothers' Social Skills, Attention Switching, Communication, and Imagination subscale scores showed weak but significant positive correlations with each subscale score on the PNQ (Table 2). However, Attention to Detail scores for both fathers and mothers were not positively correlated with the parenting difficulties subscale scores. Fathers' Attention to Detail scores showed a weak but significant negative correlation with difficulty scores for Attention/Connection and Spontaneity, and mothers' Attention to Detail scores showed a weak but significant negative correlation with all subscale scores on the PNQ except Affection.

\section{2. $t$-Tests Comparing High and Not High Autistic Traits Group}

A series of $t$-tests were performed to examine differences in parenting difficulties between parents with high and not high autistic traits. Prior to the analyses, two subgroups of parents (high and not high autistic traits groups) were created. A total AQ score of 26 or above denotes high autistic traits (Woodbury-Smith et al., 2005). The high autistic traits group comprised 91 fathers and 147 mothers, whereas the not high autistic traits group comprised 337 fathers and 798 mothers. Results showed that both fathers and mothers in the high autistic traits group reported more parenting difficulties across all subscales of PNQ (Table 3).

\subsection{Hierarchical Multiple Regression Analyses}

Hierarchical multiple regression analyses were performed to investigate which parental autistic trait domains predicted parenting difficulties after controlling for children's sex, age, and TDS. Multicollinearity was determined by reviewing variance inflation factor (VIF) statistics. A VIF value greater than 10 is generally known to indicate the presence of severe multicollinearity 
(Chatterjee \& Price, 1990; O’Brien, 2007). The VIF for all predictors was less than 1.92 in this study, indicating no multicollinearity problem. The moderate to strong significant correlations between all PNQ subscale scores and the results of the $t$-tests suggest that it is reasonable to use the total score of PNQ as the dependent variable in the hierarchical multiple regression. In the models predicting fathers' parenting difficulties, the coefficients of determination increased significantly when each subscale score of paternal autistic traits was included in the analysis in Step 2 (Table 4). Children's, sex, age, and TDS explained only $2 \%$ of the variance in the PNQ total score of fathers, whereas after entering fathers' autistic traits in Step 2, the overall model explained $13 \%$ of the variance, with the paternal autistic traits uniquely explaining an additional $11 \%$ of the variance in the PNQ total score of fathers. In Step 2, only the communication subscale score was a significant predictor of paternal parenting difficulties, suggesting that traits of impaired communication in fathers are related to difficulties in parenting.

In the models predicting mothers' parenting difficulties, a significant increase in the coefficients of determination was confirmed when each subscale score of maternal autistic traits was included in Step 2 of the analysis (Table 4). Children's sex, age, and TDS explained 11\% of the variance in the total score of the PNQ of mothers, whereas after entering mothers' autistic traits in Step 2, the model explained $20 \%$ of the variance, and the autistic traits of mothers uniquely explained an additional 9\% of the variance in the PNQ total score of mothers. In Step 2, Attention Switching, Attention to Detail, Communication, and Imagination subscale scores, in addition to children's age and TDS, were significant predictors of maternal parenting difficulties. Within each domain of maternal autistic traits, poor Attention Switching, impaired Communication, and lack of Imagination were associated with increased parenting difficulties, while higher Attention to Detail was related to lower parenting difficulties in mothers. Furthermore, younger age and higher TDS 
of children were also shown to predict parenting difficulties in mothers. Models including all the factors that contribute to parenting difficulties revealed by these hierarchical multiple regression analyses are shown separately for fathers and mothers in Figures 1 and 2, respectively.

\section{Discussion}

The purpose of the present study was to investigate the association between parental autistic traits and parenting difficulties in Japanese community samples. Results indicated that parental autistic traits were associated with parenting difficulties, and parents with higher scores for autistic traits had more difficulties in various domains of parenting. Furthermore, considering each domain of parental autistic traits, paternal traits of impaired communication related to parenting difficulties, even after controlling for children's sex, age, and total difficulties. For mothers, poor attention switching, impaired communication, and lack of imagination were associated with parenting difficulties even after controlling for children's sex, age, and total difficulties. However, higher attention to detail, one of the characteristics of ASD, was related to fewer parenting difficulties in mothers.

In the correlation analyses, both fathers and mothers with higher total scores for autistic traits had more parenting difficulties across all domains, consistent with the results showing a positive correlation between parental autistic traits and parenting difficulties (Dissanayake et al., 2020). It is worth noting that, when examined for each domain of autistic traits, Social Skills, Attention Switching, Communication, and Imagination showed the same correlations as the Total Scores; only Attention to Detail showed different results. Parental Attention to Detail did not positively correlate with parenting difficulties; it showed a weak but significant negative correlation with some subscales of parenting difficulties, especially for mothers. Excessive attention to detail is often thought to be a negative factor for social adjustment, but in parenting, 
which requires careful attention to children and the environment surrounding them changing with every moment, it may not always have a negative influence.

When comparing parenting difficulties between the high and not high autistic traits groups, fathers and mothers in the high autistic traits group had higher levels of difficulties in all domains of parenting. It is thus important to provide appropriate support for Japanese parents with higher levels of autistic traits who have preschool and/or elementary school level children not only in limited aspects of parenting but in various domains as a whole. This result is partly different from the findings of Dissanayake et al. (2020), who showed that parents with high autistic traits were more likely to have difficulties in parenting in domains other than affection and danger awareness. Differences in age of children $\left(M_{\text {age }}=10.10\right.$ years in Dissanayake et al., $2020 ; 85.12$ months in this study) or cultures may have an effect, although it is not possible to completely identify the reason. Future studies should investigate the reasons for these cultural differences.

The most notable finding of this study was that some domains of parental autistic traits predicted parenting difficulties even after controlling for children's sex, age, and TDS. The results suggest that parents with higher levels of autistic traits are more likely to face difficulties in parenting, regardless of their children's sex, age, or degree of emotional/behavioral problems. Among the various domains of autistic traits, impaired communication, the core symptom of ASD, predicted parenting difficulties for both fathers and mothers. Seay et al. (2014) reviewed studies on positive parenting and reported that communication with children involving verbalization (Gavin et al., 2002), active listening (Duncan et al., 2009), and respect (Nadeem et al., 2007; Schor \& American Academy of Pediatrics Task Force on the Family, 2003) is a factor that constitute the concept of positive parenting. Impaired communication in parents with higher levels of autistic traits impedes smooth and appropriate communication with their children in accordance with the 
situation, which can lead to parenting difficulties. In addition to communication problems, mothers' poor attention switching and a lack of imagination also predicted mothers' difficulties in parenting. In Japan, the gender gap in childcare engagement is striking, and mothers spend much more time with their children, particularly with preschool children than fathers (Gender Equality Bureau, Cabinet Office, 2020). Since mothers, with increased opportunities to interact with their children than fathers, are required to have various parenting skills, higher levels of autistic traits may lead to more parenting difficulties for mothers than fathers. Mothers are often required to multitask, such as doing household chores while caring for their children. The traits contained in the subscale of poor Attention Switching, such as being unable to do more than one thing at once easily or frequently getting strongly absorbed in one thing, might lead to difficulties in parenting for mothers. Moreover, the Imagination subscale involves the traits of understanding easily what a person is thinking or feeling just by looking at the other person's face, and if mothers are not good at this, they may not be able to properly feel the emotions or needs of their children, which can cause difficulties in parenting. It is also interesting that the regression coefficient for fathers' Attention to Detail subscale scores was not predicting parenting difficulties scores was not significant, while mothers' scores were. More notably, in contrast to our expectation, this regression coefficient was negative rather than positive.

Excessive attention to detail is commonly known as one of the characteristics of people with ASD. This trait sometimes leads to difficulties with one's social life, but it was suggested that it might be a strength of the mother with higher levels of autistic traits in parenting children. Given that parental inattention has been shown to be associated with negative parenting behavior, such as inconsistent discipline and lower involvement (Chen \& Johnston, 2007), parental traits of paying attention to details may not be considered inappropriate for parenting. leads to difficulties with 
one's social life

\subsection{Clinical Implications}

The finding that higher levels of parental autistic traits are related to parenting difficulties has clinical implications. First, the results obtained from this study suggest that it may be beneficial to assess autistic traits in fathers and mothers to identify those parents who are at a greater risk of having severe parenting difficulties. Parenting difficulties are a major source of stress for parents (Vondra \& Belsky, 1993), and parenting stress is related to negative outcomes in children, such as low social competence and high internalizing and externalizing behaviors (Anthony et al., 2005). Providing appropriate support to eliminate parenting difficulties for parents with higher levels of autistic traits is essential to prevent increased parental stress and maintain mental health and social adjustment in children. In Japan, health examinations are conducted by local governments for preschoolers and elementary school students, but there is generally no opportunity to screen their parents' behavioral characteristics or mental health. Early identification and follow-up of parents with high autistic traits might be effective in the prevention and early detection of parenting difficulties and in providing parenting support that addresses their needs, such as programs to develop communication skills and training to switch attention and improve imagination. Furthermore, it has been shown that not all domains of parental autistic traits have a negative impact on parenting; rather, higher attention to detail can sometimes prove to be a strength for parents with higher levels of autistic traits. It would be desirable to provide parenting support to address the problems caused by parental higher levels of autistic traits by making the best use of these traits as strengths for parents with higher levels of autistic traits.

Clinicians or specialists in parenting support should pay attention to autistic traits in parents with parenting difficulties even if their chief complaint is not associated with ASD because 
they may not be aware that autistic traits could be the reason for their parenting difficulties. It is not rare for people with ASD to remain undiagnosed or misdiagnosed until adulthood because of their coping and camouflaging strategies or their autistic traits being masked by other psychopathological symptoms, especially in people without intellectual disability (Fusar-Poli et al., 2020; Happé et al., 2016). It cannot be denied that fathers and mothers who have not been diagnosed with ASD or have been diagnosed with other psychiatric disorders may actually have clinical or subthreshold levels of autistic traits. Clinicians or specialists should be aware that higher levels of autistic traits may underlie parenting difficulties.

\subsection{Limitations and Future Research}

To the best of our knowledge, this is the first study to examine the association between each domain of parental autistic traits and parenting difficulties; however, there are several limitations to this study that need to be acknowledged. First, parental psychiatric symptoms that could impact parenting difficulties were not included in the analyses in this study. For example, parental depression or ADHD symptoms have been reported to affect parenting behaviors (Lovejoy et al., 2000; Park et al., 2017; Wilson \& Durbin, 2010), but the current study could not measure parental psychiatric symptoms. Given the high risk of psychiatric comorbidity in adults with ASD (Joshi et al., 2013), higher levels of autistic traits, even at the subthreshold level, can be accompanied by various psychiatric symptoms. Moreover, psychiatric symptoms and somatic conditions, such as the presence of physical illness, can be confounding factors. Another limitation of this study is that the impact of family socioeconomic status, reported as one of the factors related to parenting (Hoff-Ginsberg \& Tardif, 1995), could not be considered. Future studies should examine the association between parental autistic traits and parenting after controlling for other factors related to parenting behaviors, such as parental psychiatric symptoms, somatic conditions, family 
socioeconomic status, and more detailed family information. Furthermore, the participants in the community sample of this study were limited to four prefectures in Japan, which included both urban and rural areas. Further research needs to be conducted to determine the generalizability of the results of this study. In addition, it should be noted that this study followed a cross-sectional research design and cannot identify a causal relationship. A previous study examining the cross-sectional relationship between parental autistic traits and parenting difficulties (Dissanayake et al., 2020) stated that the risk of misinterpreting the relevant directions between these variables was quite small since it was unlikely that the parenting difficulties caused parental increased autistic traits. Longitudinal studies need to be conducted to identify the causal relationship between parental autistic traits and parenting difficulties. In addition, the current study did not identify mechanisms for the association between parental autistic traits and parenting difficulties, such as mediator or moderator variables, so future study needs to clarify such variables.

In conclusion, this study demonstrated an association between parental autistic traits and parenting difficulties in a Japanese community sample. More specifically, paternal traits of impaired communication and maternal traits of poor attention switching, impaired communication, and lack of imagination predicted parenting difficulties after controlling for children's sex, age, and emotional/behavioral problems. This highlights the importance of focusing on parents with higher levels of autistic traits even below the diagnostic subthreshold to prevent or detect early parenting difficulties and provide appropriate support to promote social adaptation and the mental health of families. 


\section{References}

Adachi, J., Yukihiro, R., \& Inoue, M. (2008). [Reliability and validity of short version of Pervasive Developmental Disorders Autism Society Japan Rating Scale (PARS); a behavior checklist for people with PDD]. Seishin Igaku, 50(5), 431-438.

American Psychiatric Association (2013). Diagnostic and statistical manual of mental disorders (5th ed.). https://doi.org/10.1176/appi.books.9780890425596

Anthony, L. G., Anthony, B. J., Glanville, D. N., Naiman, D. Q., Waanders, C., \& Shaffer, S. (2005). The relationships between parenting stress, parenting behaviour and preschoolers' social competence and behaviour problems in the classroom. Infant and Child Development, 14(2), 133-154. https://doi.org/10.1002/icd.385

Baron-Cohen, S., Wheelwright, S., Skinner, R., Martin, J., \& Clubley, E. (2001). The Autism-Spectrum Quotient (AQ): Evidence from Asperger syndrome/high-functioning autism, males and females, scientists and mathematicians. Journal of Autism and Developmental Disorders, 31(1), 5-17. https://doi.org/10.1023/A:1005653411471

Baumrind, D. (1973). The development of instrumental competence through socialization. In Minnesota Symposium on Child Psychology (Vol. 7, pp. 3-46). University of Minnesota Press. https://doi.org/10.5749/j.ctttsmk0.4

Bettelheim, B. (1967). The empty fortress: Infantile autism and the birth of the self. Free Press Brugha, T. S., Spiers, N., Bankart, J., Cooper, S. A., McManus, S., Scott, F. J., Smith, J., \& Tyrer, F. (2016). Epidemiology of autism in adults across age groups and ability levels. The British Journal of Psychiatry, 209(6), 498-503. https://doi.org/10.1192/bjp.bp.115.174649

Chatterjee, S., \& Price, B. (1990). Regression Diagnostics. Wiley.

Chen, M., \& Johnston, C. (2007). Maternal inattention and impulsivity and parenting behaviors. 
Journal of Clinical Child and Adolescent Psychology, 36(3), 455-468.

https://doi.org/10.1080/15374410701448570

Constantino, J. N., \& Charman, T. (2016). Diagnosis of autism spectrum disorder: Reconciling the syndrome, its diverse origins, and variation in expression. The Lancet. Neurology, 15(3), 279-291. https://doi.org/10.1016/S1474-4422(15)00151-9

Dissanayake, C., Richdale, A., Kolivas, N., \& Pamment, L. (2020). An exploratory study of autism traits and parenting. Journal of Autism and Developmental Disorders, 50(7), 2593-2606. https://doi.org/10.1007/s10803-019-03984-4

Duncan, L. G., Coatsworth, J. D., \& Greenberg, M. T. (2009). A model of mindful parenting: Implications for parent-child relationships and prevention research. Clinical Child and Family Psychology Review, 12(3), 255-270. https://doi.org/10.1007/s10567-009-0046-3

Fujiwara, T., Kasahara, M., Tsujii, H., \& Okuyama, M. (2014). Association of maternal developmental disorder traits with child mistreatment: A prospective study in Japan. Child Abuse and Neglect, 38(8), 1283-1289. https://doi.org/10.1016/j.chiabu.2014.04.007

Fusar-Poli, L., Brondino, N., Politi, P., \& Aguglia, E. (2020). Missed diagnoses and misdiagnoses of adults with autism spectrum disorder. European Archives of Psychiatry and Clinical Neuroscience, Advance online publication. https://doi.org/10.1016/j.chiabu.2014.04.007

Gavin, L. E., Black, M. M., Minor, S., Abel, Y., Papas, M. A., \& Bentley, M. E. (2002). Young, disadvantaged fathers' involvement with their infants: An ecological perspective. Journal of Adolescent Health, 31(3), 266-276. https://doi.org/10.1016/S1054-139X(02)00366-X

Gender Equality Bureau, Cabinet Office. (2020, July). White paper on Gender Equality, 2020: Summary. Cabinet Office, Government of Japan. https://www.gender.go.jp/english_contents/about_danjo/whitepaper/pdf/ewp2020.pdf 
Goodman, R. (1997). The Strengths and Difficulties Questionnaire: A research note. Journal of Child Psychology and Psychiatry, and Allied Disciplines, 38(5), 581-586. https://doi.org/10.1111/j.1469-7610.1997.tb01545.x

Goodman, R. (2001). Psychometric properties of the strengths and difficulties questionnaire. Journal of the American Academy of Child and Adolescent Psychiatry, 40(11), 1337-1345. https://doi.org/10.1097/00004583-200111000-00015

Happé, F. G., Mansour, H., Barrett, P., Brown, T., Abbott, P., \& Charlton, R. A. (2016). Demographic and cognitive profile of individuals seeking a diagnosis of autism spectrum disorder in adulthood. Journal of Autism and Developmental Disorders, 46(11), 3469-3480. https://doi.org/10.1007/s10803-016-2886-2

Hoff-Ginsberg, E., \& Tardif, T. (1995). Socioeconomic status and parenting. In M. H. Bornstein (Ed.), Handbook of parenting, Vol 2. Biology and ecology of parenting (pp. 161-188). Lawrence Erlbaum Associates, Inc.

Howlin, P., Goode, S., Hutton, J., \& Rutter, M. (2004). Adult outcome for children with autism. Journal of Child Psychology and Psychiatry, and Allied Disciplines, 45(2), 212-229. https://doi.org/10.1111/j.1469-7610.2004.00215.x

Hurst, R. M., Mitchell, J. T., Kimbrel, N. A., Kwapil, T. K., \& Nelson-Gray, R. O. (2007). Examination of the reliability and factor structure of the Autism Spectrum Quotient (AQ) in a non-clinical sample. Personality and Individual Differences, 43(7), 1938-1949. https://doi.org/10.1016/j.paid.2007.06.012

Joshi, G., Wozniak, J., Petty, C., Martelon, M. K., Fried, R., Bolfek, A., Kotte, A., Stevens, J., Furtak, S. L., Bourgeois, M., Caruso, J., Caron, A., \& Biederman, J. (2013). Psychiatric comorbidity and functioning in a clinically referred population of adults with autism 
spectrum disorders: A comparative study. Journal of Autism and Developmental Disorders, 43(6), 1314-1325. https://doi.org/10.1007/s10803-012-1679-5

Kamio, Y., Inada, N., Moriwaki, A., Kuroda, M., Koyama, T., Tsujii, H., Kawakubo, Y., Kuwabara, H., Tsuchiya, K. J., Uno, Y., \& Constantino, J. N. (2013). Quantitative autistic traits ascertained in a national survey of 22529 Japanese schoolchildren. Acta Psychiatrica Scandinavica, 128(1), 45-53. https://doi.org/10.1111/acps.12034

Lau, W. Y. P., Peterson, C. C., Attwood, T., Garnett, M. S., \& Kelly, A. B. (2016). Parents on the autism continuum: Links with parenting efficacy. Research in Autism Spectrum Disorders, 26, 57-64. https://doi.org/10.1016/j.rasd.2016.02.007

Lovejoy, M. C., Graczyk, P. A., O’Hare, E., \& Neuman, G. (2000). Maternal depression and parenting behavior: A meta-analytic review. Clinical Psychology Review, 20(5), 561-592. https://doi.org/10.1016/S0272-7358(98)00100-7

Matsuishi, T., Nagano, M., Araki, Y., Tanaka, Y., Iwasaki, M., Yamashita, Y., Nagamitsu, S., Iizuka, C., Ohya, T., Shibuya, K., Hara, M., Matsuda, K., Tsuda, A., \& Kakuma, T. (2008). Scale properties of the Japanese version of the Strengths and Difficulties Questionnaire (SDQ): A study of infant and school children in community samples. Brain and Development, 30(6), 410-415. https://doi.org/10.1016/j.braindev.2007.12.003

McSherry, D., Fargas Malet, M., \& Weatherall, K. (2019). The Strengths and Difficulties Questionnaire (SDQ): A proxy measure of parenting stress. The British Journal of Social Work, 49(1), 96-115. https://doi.org/10.1093/bjsw/bcy021

Moriwaki, A., \& Kamio, Y. (2014). Normative data and psychometric properties of the strengths and Difficulties questionnaire among Japanese school-aged children. Child and Adolescent Psychiatry and Mental Health, 8(1), Article 1. https://doi.org/10.1186/1753-2000-8-1 
Nadeem, E., Romo, L. F., Sigman, M., Lefkowitz, E. S., \& Au, T. K. (2007). The validity of observational measures in detecting optimal maternal communication styles: Evidence from European Americans and Latinos. Journal of Research on Adolescence, 17(1), 153-168. https://doi.org/10.1111/j.1532-7795.2007.00516.x

O'Brien, R. M. (2007). A caution regarding rules of thumb for variance inflation factors. Quality and Quantity, 41(5), 673-690. https://doi.org/10.1007/s11135-006-9018-6

Park, J. L., Hudec, K. L., \& Johnston, C. (2017). Parental ADHD symptoms and parenting behaviors: A meta-analytic review. Clinical Psychology Review, 56, 25-39. https://doi.org/10.1016/j.cpr.2017.05.003

Pisula, E., Kawa, R., Szostakiewicz, Ł., Łucka, I., Kawa, M., \& Rynkiewicz, A. (2013). Autistic traits in male and female students and individuals with high functioning autism spectrum disorders measured by the Polish version of the Autism-Spectrum Quotient. PloS One, 8(9), e75236. https://doi.org/10.1371/journal.pone.0075236

Ruta, L., Mazzone, D., Mazzone, L., Wheelwright, S., \& Baron-Cohen, S. (2012). The Autism-Spectrum Quotient-Italian version: A cross-cultural confirmation of the broader autism phenotype. Journal of Autism and Developmental Disorders, 42(4), 625-633. https://doi.org/10.1007/s10803-011-1290-1

Schor, E. L., \& the American Academy of Pediatrics Task Force on the Family (2003). Family pediatrics: Report of the task force on the family. Pediatrics, 111(6, Pt 2), 1541-1571.

Seay, A., Freysteinson, W. M., \& McFarlane, J. (2014). Positive parenting. Nursing Forum, 49(3), 200-208. https://doi.org/10.1111/nuf.12093

Tachibana, Y., Takehara, K., Kakee, N., Mikami, M., Inoue, E., Mori, R., Ota, E., Koizumi, T., Okuyama, M., \& Kubo, T. (2017). Maternal impulse control disability and developmental 
disorder traits are risk factors for child maltreatment. Scientific Reports, 7, Article 15565. https://doi.org/10.1038/s41598-017-14666-5

Takei, R., Matsuo, J., Takahashi, H., Uchiyama, T., Kunugi, H., \& Kamio, Y. (2014). Verification of the utility of the social responsiveness scale for adults in non-clinical and clinical adult populations in Japan. BMC Psychiatry, 14, Article 302.

https://doi.org/10.1186/s12888-014-0302-z

van Steijn, D. J., Oerlemans, A. M., de Ruiter, S. W., van Aken, M. A., Buitelaar, J. K., \& Rommelse, N. N. (2013). Are parental autism spectrum disorder and/or attention-deficit/Hyperactivity disorder symptoms related to parenting styles in families with ASD (+ ADHD) affected children? European Child and Adolescent Psychiatry, 22(11), 671-681. https://doi.org/10.1007/s00787-013-0408-8

van Steijn, D. J., Oerlemans, A. M., van Aken, M. A., Buitelaar, J. K., \& Rommelse, N. N. (2013). Match or mismatch? Influence of parental and offspring ASD and ADHD symptoms on the parent-child relationship. Journal of Autism and Developmental Disorders, 43(8), 19351945. https://doi.org/10.1007/s10803-012-1746-y

Vondra, J., \& Belsky, J. (1993). Developmental origins of parenting: Personality and relationship factors. In T. Luster \& L. Okagaki (Eds.), Parenting: An ecological perspective (pp. 1-33). Elbaum.

Wakabayashi, A., Tojo, Y., Baron-Cohen, S., \& Wheelwright, S. (2004). [The Autism-Spectrum Quotient (AQ) Japanese version: Evidence from high-functioning clinical group and normal adults]. Shinrigaku Kenkyu, 75(1), 78-84. https://doi.org/10.4992/jjpsy.75.78

Wilson, S., \& Durbin, C. E. (2010). Effects of paternal depression on fathers' parenting behaviors: A meta-analytic review. Clinical Psychology Review, 30(2), 167-180. 
https://doi.org/10.1016/j.cpr.2009.10.007

Woodbury-Smith, M. R., Robinson, J., Wheelwright, S., \& Baron-Cohen, S. (2005). Screening adults for Asperger syndrome using the AQ: A preliminary study of its diagnostic validity in clinical practice. Journal of Autism and Developmental Disorders, 35(3), 331-335. https://doi.org/10.1007/s10803-005-3300-7 
Table 1.

Descriptive Statistics of Variables and Characteristics of Children

\begin{tabular}{|c|c|c|c|c|c|c|}
\hline \multirow[b]{3}{*}{ Parental autistic traits (AQ) } & \multicolumn{2}{|c|}{$M(S D)$} & Range & \multicolumn{2}{|c|}{$M(S D)$} & Range \\
\hline & \multicolumn{3}{|c|}{ Fathers } & \multicolumn{3}{|c|}{ Mothers } \\
\hline & & & & & & \\
\hline Total Score & 19.86 & $(6.44)$ & $5-41$ & 17.94 & $(6.81)$ & $3-37$ \\
\hline Social Skills & 4.65 & $(2.73)$ & $0-10$ & 4.44 & $(2.66)$ & $0-10$ \\
\hline Attention Switching & 4.18 & $(2.02)$ & $0-10$ & 3.94 & $(2.01)$ & $0-10$ \\
\hline Attention to Detail & 4.53 & $(2.30)$ & $0-10$ & 4.35 & $(2.15)$ & $0-10$ \\
\hline Communication & 2.83 & $(2.06)$ & $0-9$ & 2.55 & $(2.07)$ & $0-9$ \\
\hline Imagination & 3.66 & $(1.88)$ & $0-10$ & 2.66 & $(1.74)$ & $0-9$ \\
\hline \multicolumn{7}{|l|}{ Parenting difficulties (PNQ) } \\
\hline \multirow[t]{2}{*}{ Total Score } & 48.67 & $(19.40$ & $0-132$ & 45.29 & $(18.42$ & $0-112$ \\
\hline & \multicolumn{3}{|c|}{ ) } & \multicolumn{3}{|c|}{ ) } \\
\hline Modeling/Teaching Behaviors & 5.86 & $(2.90)$ & $0-15$ & 5.31 & $(2.75)$ & $0-15$ \\
\hline Understanding Needs & 5.22 & $(2.51)$ & $0-15$ & 4.23 & $(2.30)$ & $0-14$ \\
\hline Affection & 4.17 & $(2.63)$ & $0-15$ & 3.46 & $(2.52)$ & $0-14$ \\
\hline Emotion Control & 7.42 & $(2.96)$ & $0-15$ & 7.97 & $(2.78)$ & $0-15$ \\
\hline Attention/Connection & 5.26 & $(2.37)$ & $0-15$ & 5.62 & $(2.40)$ & $0-14$ \\
\hline Spontaneity & 5.89 & $(2.61)$ & $0-15$ & 5.04 & $(2.48)$ & $0-15$ \\
\hline Communication & 5.06 & $(2.44)$ & $0-15$ & 4.22 & $(2.30)$ & $0-13$ \\
\hline Danger Awareness & 4.68 & $(2.82)$ & $0-15$ & 4.44 & $(2.69)$ & $0-14$ \\
\hline Sensory Issues & 5.10 & $(2.30)$ & $0-12$ & 4.99 & $(2.30)$ & $0-12$ \\
\hline
\end{tabular}


Sex

Boys: $50.9 \%$; Girls: $49.1 \%$

Age (in months), $M(S D)$, range

85.12 (34.16), 24-154

Emotional/Behavioral problems in children, $M$

$10.00(5.43), 0-30$

$(S D)$, range

Note. $M=$ mean; $S D=$ standard deviation; $\mathrm{AQ}=$ autism spectrum quotient; $\mathrm{PNQ}=$ parenting needs questionnaire 
Table 2.

Correlations Between Parental Autistic Traits and Parenting Difficulties

Total Score Social Skills Attention Switching Attention to Detail Communication Imagination

\begin{tabular}{|c|c|c|c|c|c|c|}
\hline \multicolumn{7}{|l|}{ Parenting difficulties (PNQ) } \\
\hline Total Score & $.29^{* * *} / .30^{* * *}$ & $.21^{* * *} / .25^{* * *}$ & $.25^{* * *} / .25^{* * *}$ & $-.09 /-.11^{* *}$ & $.31^{* * *} / .33^{* * *}$ & $.19^{* * *} / .24^{* * *}$ \\
\hline Modeling/Teaching Behaviors & $.26^{* * *} / .27^{* * *}$ & $.20^{* * *} / .23^{* * *}$ & $.20^{* * *} / .19^{* * *}$ & $-.08 /-.09^{*}$ & $.30^{* * *} / .31^{* * *}$ & $.15^{* * *} / .20^{* * *}$ \\
\hline Understanding Needs & $.24^{* * *} / .30^{* * *}$ & $.16^{* *} / .26^{* * *}$ & $.20^{* * *} / .24^{* * *}$ & $-.07 /-.08^{*}$ & $.27^{* * *} / .31^{* * *}$ & $.17^{* * *} / .23^{* * *}$ \\
\hline Affection & $.25^{* * *} / .22^{* * *}$ & $.19^{* * *} / .16^{* * *}$ & $.12 \% .15^{* * *}$ & $-.03 /-.05$ & $.25^{* * *} / .23^{* * *}$ & $.20^{* * *} / .22^{* * *}$ \\
\hline Emotion Control & $.24^{* * *} / .20^{* * *}$ & $.14^{* *} / .17^{* * *}$ & $.25^{* * *} / .21^{* * *}$ & $-.01 /-.08^{*}$ & $.21^{* * *} / .23^{* * *}$ & $.14^{* * *} / .10^{* * *}$ \\
\hline Attention/Connection & $.16^{* *} / .25^{* * *}$ & $.16^{* *} / .22^{* * *}$ & $.17^{* * *} / .21^{* * *}$ & $-.13^{* *} /-.10^{* *}$ & $.17^{* * *} / .25^{* * *}$ & $.11^{* * *} / .21^{* * *}$ \\
\hline Spontaneity & $.26^{* * *} / .28^{* * *}$ & $.21^{* * *} / .24^{* * *}$ & $.22^{* * *} / .28^{* * *}$ & $-.14^{* *} /-.12^{* * *}$ & $.29^{* * *} / .30^{* * *}$ & $.20^{* * *} / .20^{* * *}$ \\
\hline Communication & $.29^{* * *} / .26^{* * *}$ & $.20^{* * *} / .22^{* * *}$ & $.24^{* * *} / .19^{* * *}$ & $-.06 /-.09^{* *}$ & $.30^{* * *} / .28^{* * *}$ & $.18^{* * *} / .25^{* * *}$ \\
\hline Danger Awareness & $.23^{* * *} / .20^{* * *}$ & $.17^{* * *} / .17^{* * *}$ & $.21^{* * *} / .17^{* * *}$ & $-.09 /-.10^{* *}$ & $.29^{* * *} / .24^{* * *}$ & $.11^{* * *} / .18^{* * *}$ \\
\hline Sensory Issues & $.22^{* * *} / .26^{* * *}$ & $.17^{* *} / .20^{* * *}$ & $.20^{* * *} / .25^{* * *}$ & $-.09 /-.07^{*}$ & $.24^{* * *} / .28^{* * *}$ & $.12^{* * *} / .17^{* * *}$ \\
\hline
\end{tabular}

Note. The number on the left shows the value for fathers, and the number on the right shows the value for mothers. AQ $=$ autism spectrum quotient; PNQ $=$ parenting needs questionnaire; ${ }^{*} p<.05 ; * * p<.01 ; * * *<.001$ 
Table 3.

Differences in Parenting Difficulties Scores Between High and Not High Autistic Traits Groups

\begin{tabular}{|c|c|c|c|c|c|c|}
\hline & \multicolumn{3}{|c|}{ Fathers } & \multicolumn{3}{|c|}{ Mothers } \\
\hline & High Autistic & Not High Autistic & $t$ & High Autistic & Not High Autistic & $t$ \\
\hline & Traits & Traits & & Traits & Traits & \\
\hline & $(n=91)$ & $(n=337)$ & & $(n=147)$ & $(n=798)$ & \\
\hline & $M(S D)$ & $M(S D)$ & & $M(S D)$ & $M(S D)$ & \\
\hline PNQ Total Score & $58.03(17.88)$ & $46.14(19.03)$ & $5.36^{* * *}$ & $56.63(18.79)$ & $43.19(17.57)$ & $8.43^{* * *}$ \\
\hline Modeling/Teaching Behaviors & $6.98(2.86)$ & $5.55(2.84)$ & $4.24^{* * *}$ & $6.74(2.76)$ & $5.05(2.67)$ & $7.01^{* * *}$ \\
\hline Understanding Needs & $6.22(2.42)$ & $4.95(2.46)$ & $4.39^{* * *}$ & $5.55(2.46)$ & $3.99(2.18)$ & $7.83^{* * *}$ \\
\hline Affection & $5.19(2.61)$ & $3.90(2.57)$ & $4.22^{* * *}$ & $4.56(2.77)$ & $3.26(2.42)$ & $5.33^{* * *}$ \\
\hline Emotion Control & $8.40(2.77)$ & $7.16(2.96)$ & $3.58^{* * *}$ & $9.09(2.66)$ & $7.76(2.75)$ & $5.40^{* * *}$ \\
\hline Attention/Connection & $5.89(2.28)$ & $5.09(2.36)$ & $2.88^{* *}$ & $6.71(2.33)$ & $5.42(2.36)$ & $6.13^{* * *}$ \\
\hline Spontaneity & $7.11(2.44)$ & $5.56(2.56)$ & $5.17^{* * *}$ & $6.47(2.58)$ & $4.78(2.38)$ & $7.82^{* * *}$ \\
\hline Communication & $6.27(2.41)$ & $4.74(2.35)$ & $5.52^{* * *}$ & $5.47(2.32)$ & $3.99(2.22)$ & $7.39^{* * *}$ \\
\hline Danger Awareness & $5.99(2.85)$ & $4.33(2.70)$ & $5.14^{* * *}$ & $5.69(2.78)$ & $4.21(2.62)$ & $6.24^{* * *}$ \\
\hline Sensory Issues & $5.99(2.18)$ & $4.86(2.29)$ & $4.21^{* * *}$ & $6.35(2.37)$ & $4.74(2.20)$ & $7.64^{* * *}$ \\
\hline
\end{tabular}

Note. A total AQ score of 26 or above denotes high autistic traits. $M=$ mean; $S D=$ standard deviation; $\mathrm{PNQ}=$ parenting needs 
questionnaire. ${ }^{* *} p<.01 ; * * * p<.001$ 
Table 4.

Results of Hierarchical Multiple Regression Analyses

\begin{tabular}{|c|c|c|c|c|c|c|c|c|c|}
\hline & & \multicolumn{4}{|c|}{$\begin{array}{l}\text { Fathers } \\
(n=428)\end{array}$} & \multicolumn{4}{|c|}{$\begin{array}{l}\text { Mothers } \\
(n=945)\end{array}$} \\
\hline & & $B[95 \% \mathrm{CI}]$ & $S E(B)$ & $\beta$ & $\Delta R^{2}$ & $B[95 \% \mathrm{CI}]$ & $S E(B)$ & $\beta$ & $\Delta R^{2}$ \\
\hline \multirow[t]{5}{*}{ Step 1} & Children's Characteristics & & & & $.02^{*}$ & & & & $.11^{* * *}$ \\
\hline & Sex & $-3.38[-6.90,0.14]$ & 1.79 & -.09 & & $-1.56[-3.68,0.55]$ & 1.08 & -.04 & \\
\hline & Age & $-0.04[-0.09,0.01]$ & 0.03 & -.08 & & $-0.05[-0.09,-0.02]$ & 0.02 & $-.10^{* *}$ & \\
\hline & Emotional/Behavioral & $0.19[-0.13,0.52]$ & 0.17 & .05 & & $0.76[0.56,0.97]$ & 0.11 & $.22^{* * *}$ & \\
\hline & Problems & & & & & & & & \\
\hline \multirow{2}{*}{\multicolumn{2}{|c|}{ Parental Autistic Traits }} & . & & & $.11^{* *}$ & & & & $.09^{* * *}$ \\
\hline & & & & & $*$ & & & & \\
\hline & Social Skills & $0.07[-.74,0.88]$ & 0.41 & .01 & & $0.32[-0.19,0.82]$ & 0.26 & .05 & \\
\hline & Attention Switching & $1.00[-0.04,2.05]$ & 0.53 & .11 & & $0.70[0.04,1.36]$ & 0.34 & $.08^{*}$ & \\
\hline & Attention to Detail & $-0.32[-1.12,0.49]$ & 0.41 & -.04 & & $-0.98[-1.48,-0.48]$ & 0.25 & $-.11^{* *}$ & \\
\hline & & & & & & & & * & \\
\hline & Communication & $2.11[1.01,3.21]$ & 0.56 & $.22^{* *}$ & & $1.21[0.50,1.91]$ & 0.36 & $.14^{* * *}$ & \\
\hline & & & & $*$ & & & & & \\
\hline
\end{tabular}




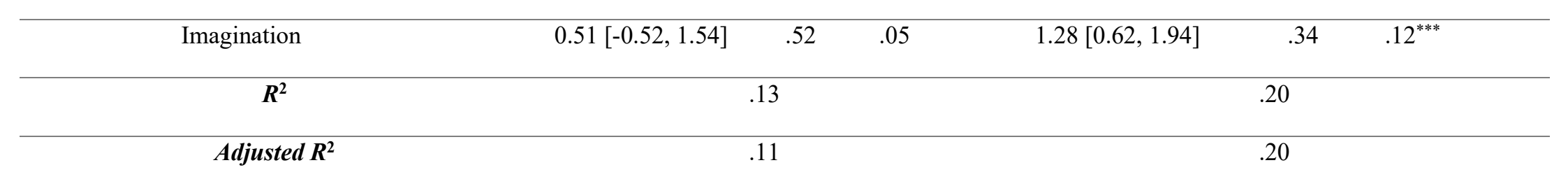

Note. $B=$ partial regression coefficient; $S E(B)=$ standard error of partial regression coefficient; $\beta=$ standardized partial regression coefficient; $R^{2}=$ coefficients of determination; adjusted $R^{2}=$ adjusted coefficients of determination; $\Delta R^{2}=$ change in coefficients of determination

$* p<.05 ; * * p<.01 ; * * *<.001$ 


\section{Figure Captions}

Figure 1. The regression model with all the factors contributing to parenting difficulties of fathers This figure shows the results of hierarchical multiple analyses of the contribution of children's characteristics (sex, age, and emotional/behavioral problems) and each domain of paternal autistic trait to the total score of parenting difficulties of fathers.

Only the variables that showed significant association are connected by arrows.

The value above the arrow indicates standardized partial regression coefficient.

Figure 2. The regression model with all the factors contributing to parenting difficulties of mothers This figure shows the results of hierarchical multiple analyses of the contribution of children's characteristics (sex, age, and emotional/behavioral problems) and each domain of maternal autistic trait to the total score of parenting difficulties of mothers.

Only the variables that showed significant association are connected by arrows.

The value above the arrow indicates standardized partial regression coefficient. 